\title{
Taxas de sucesso na aptidão física. Efeitos da idade, sexo, actividade física, sobrepeso e obesidade.
}

Passing rates on physical fitness. Effects of age, gender, physical activity, overweight and obesity.

Resumo - O principal objetivo deste estudo foi analisar a influência do género, idade, níveis de atividade física (AF) e prevalências de sobrepeso e obesidade nas taxas de sucesso global da aptidão física associada à saúde (AptFS). A amostra foi constituída por 581 meninos e 529 meninas do $1^{\circ}$ ciclo do Ensino Básico do concelho de Albergaria-a-Velha (Portugal), com idades compreendidas entre 6 e 10 anos. Foram medidos o peso e a altura e calculado o IMC. A bateria de testes do FITNESSGRAM foi aplicada. O sobrepeso e a obesidade foram determinados de acordo com os valores de corte de Cole et al. (2000). Os níveis de AF foram estimados por meio do questionário Godin \& Shephard. Registramos prevalências elevadas de sobrepeso e obesidade. Não se verificou uma associação positiva entre a AF e os níveis de AptFS. A idade não parece ser um fator de discriminação positiva ou negativa nos níveis de AptFS.

Palavras-chave: Atividade motora; Aptidão física; Criança; Sobrepeso; Obesidade.

1 Universidade do Porto. Desporto, Faculdade de Desporto. Laboratório de Cineantropometria e Estatística Aplicada. Porto. Portugal.

Recebido em 11/10/09 Revisado em 16/12/09 Aprovado 08/01/10
Abstract - The main objective of this study was to analyze the influence of gender, age, physical activity (PA) and overweight/obesity on health-related physical fitness (HRPF) passing rates. A sample of 581 boys and 529 girls, aged 6 to 10 years and enrolled in first cycle primary schools in Albergaria-a-Velha, Portugal, was studied. Weight and height were measured and BMI was calculated. The FITNESSGRAM test battery was applied. The cut-off values of Cole et al. (2000) for overweight and obesity were used. PA levels were estimated using the questionnaire of Godin and Shephard. A high prevalence of overweight and obesity was found. No positive association was observed between PA and HRPF. Age does not seem to have an impact on HRPF passing rates.

Key words: Motor activity; Physical fitness; Children; Nutritional status. 


\section{INTRODUÇÃO}

A Aptidão Física associada à saúde (AptFS) é, simultaneamente, um conceito complexo e um instrumento operacional de mensuração de um conjunto lato de atributos físicos, psicológicos e fisiológicos que cada pessoa possui em determinado momento da sua história de vida, cujos valores moderados-aelevados estão intimamente associados a um risco reduzido de desenvolvimento de doenças de natureza hipocinética ${ }^{1}$. Trata-se da capacidade de executar actividades físicas de forma enérgica e vigorosa, sem excesso de fadiga e, ao mesmo tempo, a demonstração de qualidades e capacidades que conduzam ao menor risco de desenvolvimento de doenças e incapacidades funcionais ${ }^{2}$. Com efeito, níveis moderados a elevados de componentes da AptFS como a resistência cardiovascular, a resistência e força musculares, a flexibilidade e níveis baixos de massa gorda são características do sujeito adulto associadas à sua saúde, por serem fatores de proteção de diversas doenças crônicas ligadas à morbilidade e mortalidade 3 .

A preocupação em determinar e atribuir significado pedagógico e epidemiológico aos níveis de AptFS tem conduzido a investigação a dirigir o seu olhar para populações diferenciadas em termos etários e contextuais. Por exemplo, diferentes pesquisas procuram descrever os níveis de aptidão de distintos grupos, no seio de cada população, sobretudo o comportamento em função do gênero e da idade, tentando perceber a relevância de fatores de natureza genética e/ou ambiental. DiNapoli e Lewis ${ }^{4}$, num estudo com crianças entre os 6 e 14 anos de idade, de New Hampshire, nos EUA, reportaram taxas de sucesso global de $45.4 \%$ a $63.0 \%$ nos meninos e $31.0 \%$ a $59.2 \%$ nas meninas, tendo concluído igualmente que $61.1 \%$ das crianças com 6 anos obtiveram aprovação em todas as provas e que, apenas $36.4 \%$ das crianças com 10 anos o conseguiram. No Brasil, um estudo de Aracaju ${ }^{5}$ (10-14 anos de idade) mostrou um comportamento irregular nas provas de força e flexibilidade ao longo da idade e um declínio da resistência aeróbia. No norte de Portugal, uma pesquisa (6-10 anos de idade) na cidade da Maia ${ }^{6}$ registrou taxas de sucesso, em cada prova, baixas a moderadas para ambos os sexos. Em Amarante, também no norte do país, Sousa e Maia avaliaram os níveis de aptidão física de crianças do $1^{\circ}$ Ciclo do Ensino Básico, reportando taxas de sucesso global entre os $11.3 \%$ e os $24.1 \%$ para os meninos, e os $11.4 \%$ e os $16.1 \%$ para as meninas.

Entre os fatores que influenciam os níveis de AptFS, encontram-se o sobrepeso e a obesidade, bem como os níveis de atividade física (AF). Investigações de natureza epidemiológica registraram, ainda, associações negativas entre a AptFS e o índice de massa corporal (IMC) ${ }^{4-8}$. No contexto português e não só, há evidências de que as crianças apresentam níveis preocupantes de obesidade ${ }^{7,9-11}$. No estudo com 3742 crianças, entre os 6 e os 10 anos de idade, do Arquipélago dos Açores, Maia e Lopes $^{9}$ registraram uma prevalência de sobrepeso e obesidade de, aproximadamente, $15 \%$ e $12 \%$, respectivamente. Em 2006, na cidade de Viana de Castelo, Rodrigues et al..$^{11}$ analisaram 4071 crianças da mesma faixa etária, observando valores de sobrepeso de $26 \%$ para meninos e $30.9 \%$ para meninas.

Os níveis de AF em crianças e sua associação com a AptFS não está bem estabelecida. Algumas pesquisas $s^{12-13}$ sugerem relações fracas e moderadas, se não inexistentes, tornando a natureza deste nexo relacional inconsistente. Em um estudo com crianças entre os 8 e os 11 anos, Dencker et al..$^{12}$ observaram que apenas uma pequena porção da capacidade aeróbia é explicada pelos níveis de AF. Ruiz et al. ${ }^{13}$ reportaram associações positivas entre AF intensa e gordura corporal, concluindo que a $\mathrm{AF}_{\text {total }}$ e, pelo menos, a de nível moderado, pode melhorar a aptidão cardiorrespiratória de crianças e adolescentes.

Face à inexistência de informação extensa que cubra o território português, surge a necessidade de estudar uma zona geográfica distinta das anteriormente reportadas, considerando crianças do $1^{\circ}$ Ciclo do Ensino Básico e com os seguintes objectivos: (i) descrever o comportamento das taxas de sucesso nos níveis de AptFS, ao longo da idade, das crianças de Albergaria-a-Velha entre os 6 e os 10 anos, (ii) estimar a prevalência de sobrepeso e obesidade, (iii) estimar os níveis de AF e (iv) analisar a influência dos fatores sexo, idade, níveis de AF e prevalências de sobrepeso e obesidade nas taxas de sucesso global da AptFS.

\section{PROCEDIMENTOS METODOLÓGICOS}

\section{Amostra}

A amostra deste estudo (Tabela 1) foi constituída por 1110 crianças (581 meninos e 529 meninas) entre os 6 e os 10 anos de idade, provenientes de 25 escolas (de um total de 26) do Concelho de Albergaria-a-Velha, uma vila situada na Região Central de Portugal. A amostra corresponde a $89 \%$ das crianças $(\mathrm{N}=1244)$ inscritas no $1^{\circ} \mathrm{Ciclo}$ do Ensino Básico, sendo que 36\% moram em zona urbana, 45\% em zona rural e 19\% em zona mista. 
Tabela 1. Estratificação da amostra por idade e sexo.

\begin{tabular}{cccccc}
\hline Idade & Meninos & $\%$ & Meninas & $\%$ & Total \\
\hline 6 & 100 & 17,2 & 79 & 14,9 & 175 \\
7 & 137 & 23,6 & 155 & 29,3 & 288 \\
8 & 111 & 19,1 & 115 & 21,7 & 227 \\
9 & 151 & 26,0 & 118 & 22,3 & 265 \\
10 & 84 & 14,5 & 67 & 12,7 & 155 \\
Total & 581 & & 529 & & 1110 \\
\hline
\end{tabular}

Medidas Antropométricas

A altura foi medida com um estadiômetro, sendo o resultado anotado em centímetros; o peso foi medido com balança de precisão igual a $100 \mathrm{~g}$ e o resultado expresso em quilogramas com aproximação ao hectograma. Dessas medidas, foi calculado o IMC, que corresponde à razão entre o peso e o quadrado da altura $\left(\mathrm{kg} / \mathrm{m}^{2}\right)$. Foram utilizados os valores de corte de Cole et al..$^{14}$ para definir sobrepeso e obesidade.

\section{Actividade Física}

O questionário de Godin \& Shephard ${ }^{15}$ foi adotado para recolher informação relativa à AF realizada na ocupação dos tempos livres. Esse instrumento registra o número de vezes, em média por semana, de dispêndio energético superior a quinze minutos em actividades classificadas como leves (3 Mets), moderadas (6 Mets) ou intensas/vigorosas (9 Mets). Os valores registrados são inseridos numa equação simples para estimar a $\mathrm{AF}_{\text {semanal }}$, tal que o score final é obtido através da multiplicação da frequência, em cada categoria, pelo valor do respectivo MET e pela soma posterior dos produtos obtidos, sendo:

$\mathrm{AF}_{\text {semanal }}=(9 * \mathrm{AF}$ intensa $)+\left(5^{*} \mathrm{AF}\right.$ moderada $)+\left(3^{*} \mathrm{AF}\right.$ ligeira $)$

O modo de obtenção de resposta foi por entrevista realizada pelo professor titular. Para limitar a magnitude do erro associado optou-se por: (i) entrevistar diretamente cada criança; (ii) situar as perguntas no contexto das rotinas de vida diária das crianças; (iii) recorrer ao procedimento teste-reteste a 50 crianças selecionadas aleatoriamente, que responderam ao questionário novamente, após duas semanas. As crianças foram classificadas em pouco ativas quando seu valor de $\mathrm{AF}_{\text {semanal }}$ se encontrava abaixo do percentil $33\left(\mathrm{P}_{33}\right)$, em moderadamente ativas entre o $\mathrm{P}_{33} \mathrm{e}$ $\mathrm{P}_{66}$ e em muito ativas quando se encontravam acima do $\mathrm{P}_{66}$. Os percentis foram definidos em função do género em cada valor discreto de idade.

Aptidão Física associada à Saúde

Para avaliar a AptFS foi escolhida a bateria de testes FITNESSGRAM ${ }^{16}$. É uma bateria cujos resultados são interpretados em termos criteriais, procurando mapear níveis de AptF em função da Zona Saudável de Aptidão Física (ZSAF). As crianças são classificadas como aptas ou inaptas de acordo com o sucesso ou insucesso nas provas. Foram considerados os seguintes testes: marcha/corrida da milha (1609 metros); Curl Up; Push Up a 90; e Trunk Lift. Foram considerados os valores critério da ZSAF definidos no manual da bateria ${ }^{16}$.

Procedimentos de recolha e análise dos dados Dez pessoas foram preparadas cuidadosamente, tendo sido realizada uma acção de formação teóricoprática, anterior ao trabalho de campo, para familiarizar a equipe com o protocolo de avaliação.

Foram calculadas as taxas de sucesso global da bateria Fitnessgram; o teste Qui-quadrado ( $\left.\chi^{2}\right)$ foi aplicado para pesquisar diferenças estatisticamente significativas entre valores discretos de idade, nas taxas de sucesso das provas de aptidão física e prevalências de sobrepeso e obesidade; a regressão logística foi utilizada para verificar a influência da idade, sexo, níveis de AF e prevalências de sobrepeso e obesidade nas taxas de sucesso das provas de AptFS; as prevalências de sobrepeso e obesidade e respectivos intervalos de confiança foram calculados no programa PEPI 4.0. A ANOVA a dois factores foi utilizada para testar diferenças de médias na AF. Todos os cálculos foram realizados nos softwares SPSS 16.0 e PEPI 4.0. O nível de significância foi fixado em 5\%.

Fiabilidade dos dados

Os coeficientes de correlação intraclasse (R) e os respectivos intervalos de confiança $\left(\mathrm{IC}_{95 \%}\right)$ foram utilizados para estimar quantidades de variância verdadeira por forma a obter medidas de fiabilidade. Os resultados traduzem uma fiabilidade elevada com todos os valores de $\mathrm{R}$ acima de 0.800 . Foram igualmente calculados os coeficientes de correlação canônica (Rc) para testar fiabilidade multivariada no conjunto das provas da bateria de testes do Fitnessgram. Os resultados obtidos são satisfatórios: (i) As Rc obtidas situam-se entre 0.82 e 0.99 e, (ii) o índice de redundância é de 0.920 , indicando, inequivocamente, a excelente qualidade dos dados obtidos.

\section{RESULTADOS}

\section{Aptidão Física associada à Saúde}

Os resultados relativos ao sucesso em todas as provas situam-se entre $26.6 \%$ e $38.5 \%$ para meninos, e $11.9 \%$ e 28.2\% para meninas (Tabela 2.). Não se registaram diferenças estatisticamente significativas ao longo da idade 
nos meninos $(p=0.982)$. Nas meninas essa diferença verificou-se somente entre os 6 e 10 anos $(p<0.05)$.

\section{Sobrepeso e Obesidade}

A prevalência total de sobrepeso é de $21.3 \%$ $\left(\mathrm{IC}_{95 \%}=18.1-24.9\right)$ para meninos e de $27.6 \%$ $\left(\mathrm{IC}_{95 \%}=23.8-31.6\right)$ para meninas. Os valores de obesidade são inferiores com 14.0\% $\left(\mathrm{IC}_{95 \%}=11.2-17.0\right)$ para os meninos e $12.7 \%\left(\mathrm{IC}_{95 \%}=10.0-15-8\right)$ para as meninas (Tabela 4). $\mathrm{O}$ estudo da tendência do comportamento das diferentes prevalências ao longo da idade não identificou diferenças estatisticamente significativas entre valores adjacentes de idade, à exceção dos níveis de obesidade dos meninos dos 8 para os $9(\mathrm{p}<0.05)$, e das meninas dos 7 para os 8 $(\mathrm{p}<0.05)$ e dos 8 para os 9 anos de idade $(\mathrm{p}<0.05)$. Ademais, os resultados indicam uma tendência negativa, ainda que não significativa ( $p>0.05)$, para a prevalência de normoponderais e obesos quer em meninos como em meninas, e uma tendência positiva, igualmente não significativa $(\mathrm{p}>0.05)$, para ambos os gêneros nas prevalências de sobrepeso.

\section{Actividade Física}

10 s valores médios da $\mathrm{AF}_{\text {semanal }}$ situam-se entre 69.3 e 79.5 Mets para meninos e 57.7 e 67.4 Mets para meninas (Gráfico 1). Os resultados da ANOVA a dois fatores (idade e sexo) salientaram o seguinte: (i) um efeito significativo da idade $\left(\mathrm{F}_{(4,1)}=2.687\right.$, $\mathrm{p}<0.05)$, nomeadamente entre os 7 e 10 anos $(\mathrm{p}=0.037)$; (ii) uma diferença marginal entre os 7 e 9 anos ( $p=0.083)$; (iii) um efeito significativo do sexo $\left(F_{(1,4)}=24.369, \mathrm{p}<0.001\right)$; (iv) inexistência de efeitos da interação $\left(\mathrm{F}_{(1,4)}=0.281, \mathrm{p}=0.890\right)$.

Gráfico 1. Perfil da AFsemanal entre os 6 e os 10 anos de idade para ambos os géneros.

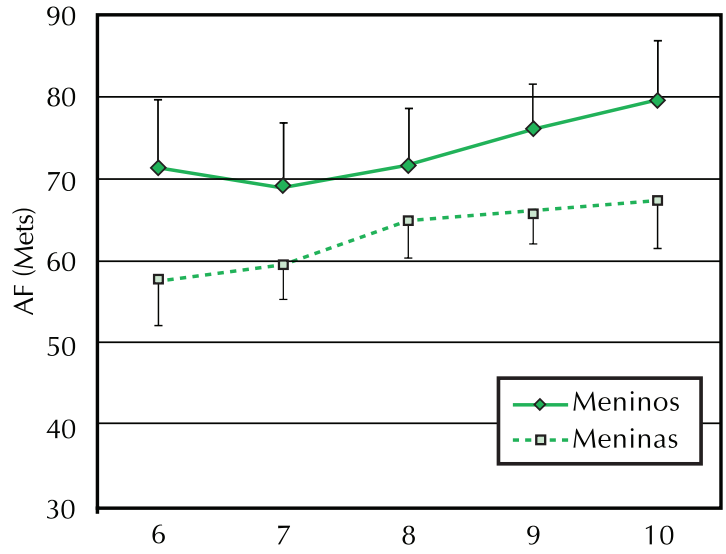

Influência dos fatores sexo, idade, níveis de AF e prevalências de sobrepeso e obesidade nas taxas de sucesso global da AptFS

Observa-se na tabela 4, que as meninas têm, aproximadamente, metade das chances dos meninos de serem aptas em todas as provas $\left(\mathrm{IC}_{95 \%}=0.36-0.63\right.$,

Tabela 2. Taxas de sucesso e respectivos intervalos de confiança a $95 \%$ em todas as provas dos meninos e meninas, ao longo da idade.

\begin{tabular}{|c|c|c|c|c|c|c|c|c|c|c|}
\hline & \multicolumn{2}{|r|}{6} & \multicolumn{2}{|c|}{7} & \multicolumn{2}{|c|}{8} & \multicolumn{2}{|c|}{9} & \multicolumn{2}{|c|}{10} \\
\hline & Mas. & Fem. & Mas. & Fem. & Mas. & Fem. & Mas. & Fem. & Mas. & Fem. \\
\hline $\begin{array}{c}\text { Conjunto } \\
\text { de provas } \\
\%\left(\mathrm{IC}_{95 \%}\right)\end{array}$ & $\begin{array}{c}27.8 \\
(19.2-37.9)\end{array}$ & $\begin{array}{c}28.2 \\
(18.6-39.5)\end{array}$ & $\begin{array}{c}35.5 \\
(27.1-44.6)\end{array}$ & $\begin{array}{c}23.3 \\
(16.8-30.9)\end{array}$ & $\begin{array}{c}36.9 \\
(28.0-46.6)\end{array}$ & $\begin{array}{c}15.5 \\
(9.5 .23 .4)\end{array}$ & $\begin{array}{c}38.5 \\
(30.6-46.9)\end{array}$ & $\begin{array}{c}15.4 \\
(9.4-23.2)\end{array}$ & $\begin{array}{c}26.6 \\
(17.3-37.1)\end{array}$ & $\begin{array}{c}11.9 \\
(4.9-22.9)\end{array}$ \\
\hline
\end{tabular}

Tabela 3. Frequências de sobrepeso e obesidade, e intervalos de confiança (IC), a 95\%.

\begin{tabular}{|c|c|c|c|c|c|c|c|c|c|c|}
\hline Idade & Sexo & \multicolumn{2}{|c|}{ Peso Adequado } & \multicolumn{3}{|c|}{ Sobrepeso } & \multicolumn{3}{|c|}{ Obesidade } & $\frac{\text { Total }}{\mathrm{N}}$ \\
\hline \multirow{2}{*}{6} & Mas & 62 & 63.9 & 18 & 18.6 & $11.4-27.7$ & 17 & 17.5 & $10.6-26.6$ & 97 \\
\hline & Fem & 48 & 61.5 & 21 & 26.9 & $17.5-38.2$ & 9 & 11.5 & $5.4-20.8$ & 78 \\
\hline 7 & Mas & 97 & 70.3 & 26 & 18.8 & $12.7-26.4$ & 15 & 10.9 & $6.2-17.3$ & 138 \\
\hline 8 & Fem & 76 & 65.5 & 34 & 29.3 & $21.2-38.5$ & 6 & 5.2 & $1.9-10.9$ & 116 \\
\hline \multirow{2}{*}{9} & Mas & 98 & 66.2 & 37 & 25.0 & $18.3-32.8$ & 13 & 8.8 & $4.8-14.6$ & 148 \\
\hline & Fem & 62 & 53.0 & 35 & 29.9 & $21.8-39.1$ & 20 & 17.1 & $10.8-25.2$ & 117 \\
\hline Total & Fem & 316 & 59.7 & 146 & 27.6 & $23.8-31.6$ & 67 & 12.7 & $10.0-15.8$ & 529 \\
\hline
\end{tabular}


Tabela 4. Resultados do modelo de regressão logística relativa à influência dos factores Sexo, Idade, IMC e AF na taxa de sucesso em todas as provas de aptidão física.

\begin{tabular}{|c|c|c|c|}
\hline Variáveis Independentes & & $\mathrm{OR}\left(\mathrm{IC}_{95 \%}\right)^{\mathrm{a}}$ & $\mathrm{p}$ \\
\hline \multirow{2}{*}{ Sexo } & Masculino $^{b}$ & 1 & \\
\hline & Feminino & $0.48(0.36-0.63)$ & $<0.001$ \\
\hline IMC Normoponderais ${ }^{b}$ & & 1 & \\
\hline IMC Sobrepeso & & $0.51(0.36-0.72)$ & $<0.001$ \\
\hline Moderadamente Activos & & $1.05(0.75-1.48)$ & 0.786 \\
\hline Muito Activos & & $1.15(0.82-1.61)$ & 0.436 \\
\hline
\end{tabular}

$\mathrm{p}<0.001)$. Crianças com sobrepeso $\left(\mathrm{IC}_{95 \%}=0.36\right.$ 0.72; $\mathrm{p}<0.01)$ e obesidade $\left(\mathrm{IC}_{95 \%}=0.12-0.37 ; \mathrm{p}<0.01\right)$ têm menor chance de obterem sucesso em todas as provas do que as normoponderais. $\mathrm{A}$ idade $\left(\mathrm{IC}_{95 \%}=\right.$ 0.814-1.007, $\mathrm{p}=0.068$ ) e as categorias distintas de AF (Moderada: $\mathrm{IC}_{95 \%}=0.745-1.475, \mathrm{p}=0.786$; Elevada: $\left.\mathrm{IC}_{95 \%}=0.818-1.610, \mathrm{p}=0.426\right)$ não são preditores significativos das taxas globais de sucesso em todas as provas de aptidão física.

\section{DISCUSSÃO}

As taxas de sucesso global da AptFS das crianças de Albergaria-a-Velha situam-se entre os $11.9 \%$ e $38.5 \%$. Estes valores são ligeiramente superiores aos registrados por Pereira ${ }^{6}$, que avaliou 793 crianças de 18 escolas do $1^{\circ}$ Ciclo do Ensino Básico da Maia, tendo obtido valores entre os 0 e $30 \%$, e inferiores aos de DiNapoli \& Lewis ${ }^{4}$, com $45.4 \%$ a $63 \%$ nos meninos e $31.0 \%$ a $59.2 \%$ nas meninas, num estudo com 6511crianças entre 6 e 14 anos de idade, de New Hampshire, nos EUA. Kim et al. ${ }^{17}$, num estudo com 6297 crianças americanas, com idades compreendidas entre os 6 e os 14 anos, reportaram taxas de sucesso de $33.7 \%$ para meninos e $31.3 \%$ para meninas. Fatores de ordem ambienta ${ }^{18}$, diferenças no estatuto socioeconômico e/ou frequências distintas de estágios maturacionais ${ }^{19}$ podem estar na origem dessas diferenças. Acrescentamos, também, a circunstância dos espaços disponíveis das escolas, os conteúdos e frequência semanal das aulas de Educação Física (EF) apoiarem a interpretação dessas diferenças. Por exemplo, Reed et al. ${ }^{20}$ concluíram que a implementação de programas efetivos de $\mathrm{EF}$ nas escolas promove um incremento substancial (20\%) nos níveis de Aptidão Aeróbia, embora haja variação significativa neste resultado.

A sociedade moderna, principalmente, a dita "industrializada", padece de um grave problema de saúde pública com origem nas elevadas taxas de so- brepeso e obesidade, e sua associação com o incremento de diversas morbilidades e mortalidade ${ }^{21,22}$. Além disso, sabe-se que há uma relação positiva entre obesidade na infância e na idade adulta. A presente pesquisa verificou que $27.6 \%$ das meninas e $21.3 \%$ dos meninos têm sobrepeso e que $12.7 \%$ das meninas e $13.9 \%$ dos meninos são obesos. Esses resultados são superiores aos registrados em outras zonas de Portugal. Padez et al. ${ }^{23}$, num estudo com crianças entre os 7 e os 9 anos de idade, da cidade de Lisboa, concluíram que $11.3 \%$ da amostra é obesa e 20.3\% tem sobrepeso. Em Vouzela, Maia et al. ${ }^{10}$ reportaram valores de obesidade entre $3.9 \%$ e $9.8 \%$ e de sobrepeso entre $15.6 \%$ e $25.0 \%$, num estudo realizado com 458 crianças do $1^{\circ}$ Ciclo do Ensino Básico. Em Amarante ${ }^{7}$, os índices de obesidade situam-se entre $4.1 \%$ e $7.2 \%$, ao passo que em Viana de Castelo, num estudo com 4701 crianças (6-10 anos), Rodrigues et al. ${ }^{11}$ relataram valores superiores de sobrepeso, com $30.9 \%$ para as meninas e $26.0 \%$ para os meninos. Diferenças marcantes no estilo de vida e hábitos nutricionais das crianças podem explicar essas diferenças. Carvalhal et al..$^{24}$ concluíram que passar mais de uma hora por dia a jogar computador resulta numa diminuição de $45 \%$ de chances dos meninos serem normoponderais. Nas meninas, a probabilidade foi ainda maior com valores na ordem dos $57 \%$. O estatuto socioeconômico e o local de residência (urbano ou rural) podem também explicar tais dados. Crianças de zonas urbanas com estatuto socioeconômico elevado tendem a ingerir maior quantidade de alimentos altamente calóricos ${ }^{25}$.

A relação positiva entre níveis moderados a elevados de AF e a saúde tem sido documentada ${ }^{26}$. Em termos médios, as crianças de Albergaria-aVelha apresentam valores de $\mathrm{AF}_{\text {total }}$ de $73.3 \mathrm{Mets} /$ semana para meninos e de 62.9 Mets/semana para meninas. Esses resultados são superiores aos relatados por Maia et al. ${ }^{10}$, que observaram valores 
entre 45.9 e 48.0 Mets/semana para os rapazes e entre 34.4 e 49.0 Mets/semana para as meninas, e inferiores aos da pesquisa Amarantina ${ }^{7}, \mathrm{com} 84.0 \mathrm{e}$ 69.7 Mets, respectivamente, para meninos e meninas. Registra-se uma tendência de crescimento dos níveis de $\mathrm{AF}$ ao longo da idade em concordância com os estudos supracitados. As diferenças nos resultados alcançados entre diferentes populações em Portugal são expectáveis, sendo diversos os fatores que as influenciam, de ordem psicológica, cultural, ambiental e social ${ }^{1}$.

Os resultados indicam uma relação positiva entre os níveis de aptidão e as prevalências de sobrepeso e obesidade. De fato, quando comparadas com crianças normoponderais, a probabilidade das crianças com sobrepeso e obesidade serem aptas é de 50\% e 20\%, respectivamente. Diversos estudos têm demonstrado essa relação. Por exemplo, Moreno et al. ${ }^{27}$ avaliaram 101 jovens espanhóis e concluíram que indivíduos com elevado nível de aptidão cardiorrespiratória apresentam menores valores de massa gorda. Huang \& Malina ${ }^{8}$, em seu estudo com 102 mil jovens de Taiwan (9-18 anos) encontraram as mesmas evidências em todas as idades e em ambos os sexos.

No que concerne à associação entre AF e AptFS, os resultados do presente estudo indicam não existir qualquer tipo de relação significativa e contrariam as evidências de outras pesquisas ${ }^{12,13}$, embora essas apenas se refiram à associação entre a $\mathrm{AF}$, aptidão aeróbia e massa gorda. A obrigatoriedade de obtenção de sucesso em todas as provas poderá explicar a divergência dos resultados obtidos. Por outro lado, existem evidências de que o padrão de AF em crianças é, muitas vezes, aleatório, esporádico e, portanto, poderá não resultar em melhorias na aptidão física ${ }^{28}$.

A associação da AptFS com a idade tem sido descrita como negativaa ${ }^{4,5,29}$, i.e., ao longo da idade, os níveis da AptFS tendem a diminuir. Os resultados deste estudo não evidenciaram esse padrão, sendo que, independentemente da idade, as crianças têm aproximadamente as mesmas chances de serem aptas em todas as provas. Não encontramos outras pesquisas com o recurso à regressão logística para testar esta associação, o que limita a comparação dos nossos resultados.

A última variável analisada foi o género. Os resultados evidenciam que a probabilidade de uma menina ser apta é aproximadamente metade da de um rapaz. Não encontramos nenhuma pesquisa com este tipo de abordagem na globalidade das provas. Dencker et al. ${ }^{30}$ encontraram diferenças na aptidão aeróbia de meninos e meninas, sendo que os rapazes apresentam valores superiores entre $8 \% \mathrm{e}$ $18 \%$. Os autores não conseguiram, no entanto, devido à natureza transversal do estudo, compreender as causas desses valores, sendo para tal necessário realizar pesquisas de natureza longitudinal.

Este estudo apresentou algumas limitações. A sua natureza transversal, de algum modo, condiciona as interpretações dos resultados obtidos ao longo do tempo, ainda que a dimensão amostral de magnitude muito elevada seja representativa de toda uma região do país. A aplicação dos questionários de AF pelo professor titular de cada turma potencia o aparecimento de algumas divergências inter-observador, não obstante o treinamento a que foram submetidos.

\section{CONCLUSÃO}

As crianças de Albergaria-a-Velha apresentam prevalências de sobrepeso e obesidade elevadas, que além das repercussões no estado futuro de saúde, são fatores de insucesso nas provas de AptFS. A AF não parece ser uma variável explicativa dos níveis de AptFS, assim como a idade não demonstra ser um fator de discriminação positiva ou negativa no sucesso em todas as provas da bateria FITNESSGRAM. Os meninos têm mais chances de serem aptos do que as meninas.

\section{REFERÊNCIAS BIBLIOGRÁFICAS}

1. Bouchard C, Sherpard RJ. Physical Activity, fitness and health: The model and key concepts. In: Bouchard C, Shepard RJ, Stevens T, editors. Physical Activity, Fitness, and Health International Proceedings and Consensus Statement. Champaign: Human Kinetics; 1994.

2. Silva R, Silva Júnior A, Cabral de Oliveira A. Crescimento em crianças e adolescentes: um estudo comparativo. Rev Bras Cineantropom Desempenho Hum 2005;7(2):55-61.

3. Lohman TG, Ring K, Pfeiffer K, Camhi S, Arredondo E, Pratt C, et al. Relationships among fitness, body composition, and physical activity. Med Sci Sports Exerc 2008 ;40(6):1163-70.

4. DiNapoli PP, Lewis JB. Understanding school-age obesity - Through participatory action research. MCN Am J Matern Child Nurs 2008;33(2):104-10.

5. Araújo SSd, Oliveira ACCd. Aptidão Física em Escolares de Aracaju. Rev Bras Cineantropom Desempenho Hum 2008;10(3):271-276.

6. Pereira AMR. Crescimento somático e aptidão física de crianças com idades compreendidas entre os 6 e os 10 anos de idade. Um estudo no concelho da Maia [Dissertação de Mestrado]. Porto: Universidade do Porto; 2000. 
7. Sousa MAC, Maia JAR. Crescimento Somático, Actividade Física e Aptidão Física Associada à Saúde - Um estudo populacional nas crianças do $1^{\circ}$ Ciclo do Ensino Básico do Concelho de Amarante. Porto: FCDEF; 2005.

8. Huang YC, Malina RM. BMI and health-related physical fitness in Taiwanese youth 9-18 years. Med Sci Sports Exerc 2007;39(4):701-8.

9. Maia JA, Lopes VP. Estudo do crescimento somático, aptidão física, actividade física e capacidade de coordenação corporal de crianças do $1^{\circ}$ Ciclo do Ensino Básico da RAA. RAA: DREFD-RAA, DRCT, FCDEF; 2002.

10. Maia JAR, Silva RGM, Seabra A. Vouzela Activo - Um olhar sobre o crescimento, o desenvolvimento e a saúde de crianças, jovens e famílias do concelho de Vouzela. Porto: FADEUP; 2009.

11. Rodrigues LP, Sá C, Bezerra P, Saraiva L. Estudo Morfofuncional da Criança Vianense. Viana do Castelo: CMVC; 2006.

12. Dencker M, Thorsson O, Karlsson MK, Linden C, Svensson J, Wollmer P, et al. Daily physical activity and its relation to aerobic fitness in children aged 8-11 years. Eur J Appl Physiol 2006;96(5):587-92.

13. Ruiz JR, Rizzo NS, Hurtig-Wennlof A, Ortega FB, Warnberg J, Sjostrom M. Relations of total physical activity and intensity to fitness and fatness in children: the European Youth Heart Study. Am J Clin Nutr 2006;84(2):299-303.

14. Cole TJ, Bellizzi MC, Flegal KM, Dietz WH. Establishing a standard definition for child overweight and obesity worldwide: international survey. BMJ 2000;6;320(7244):1240-3.

15. Godin G, Shepard RJ. A simple method to assess exercise behaviour in the comunity. Can J Appl Sport Sci1985;10:141-6.

16. Meredith MD, Welk GJ. Fitnessgram. Test administration manual. Edition n, editor. Champaign: Human Kinetics; 1999.

17. Kim J, Must A, Fitzmaurice GM, Gillman MW, Chomitz V, Kramer E, et al. Relationship of physical fitness to prevalence and incidence of overweight among schoolchildren. Obesity Res 2005;13(7):1246-54.

18. Ortega FB, Ruiz JR, Castillo MJ, Sjostrom M. Physical fitness in childhood and adolescence: a powerful marker of health. Int J Obes 2008;32(1):1-11.

19. Malina RM, Bouchard C, Bar-Or O. Growth, Maturation and Physical Activity. 2nd ed. United States: Human Kinetics; 2004.

20. Reed KE, Warburton DER, Macdonald HM, Naylor PJ, Mckay HA. Action Schools! BC: A school-based physical activity intervention designed to decrease cardiovascular disease risk factors in children. Prev Med 2008;46(6):525-31.
21. Guo SS, Wu W, Chumlea WC, Roche AF. Predicting overweight and obesity in adulthood from body mass index values in childhood and adolescence. Am J Clin Nutr $2002 ; 76(3): 653-8$.

22. Baker JL, Olsen LW, Sorensen TIA. Childhood bodymass index and the risk of coronary heart disease in adulthood. N Engl J Med 2007;357(23):2329-37.

23. Padez C, Mourao I, Moreira P, Rosado V. Prevalence and risk factors for overweight and obesity in Portuguese children. Acta Paediatrica 2005;94(11):1550-7.

24. Carvalhal MM, Padez MC, Moreira PA, Rosado VM. Overweight and obesity related to activities in Portuguese children, 7-9 years. Eur J Public Health 2007;17(1):42-6.

25. Shi Z, Lien N, Kumar BN, Holmboe-Ottesen G. Sociodemographic differences in food habits and preferences of school adolescents in Jiangsu Province, China. Eur J Clin Nutr 2005;59(12):1439-48.

26. Pate RR, O'Neill JR. Summary of the American Heart Association scientific statement: promoting physical activity in children and youth: a leadership role for schools. J Cardiovasc Nurs 2008;23(1):44-9.

27. Moreno LA, Joyanes M, Mesana MI, GonzalezGross M, Gil CM, Sarria A, et al. Harmonization of anthropometric measurements for a multicenter nutrition survey in Spanish adolescents. Nutrition 2003;19(6):481-6.

28. Rowland T. Exercise testing. In: TW R, editor. Developmental exercise physiology. Champaign: Human kinetics; 1996. p. 27-44.

29. Maia JA, Lopes VP. Um Olhar sobre crianças e jovens da Região Autónoma dos Açores: implicações para a educação física, desporto e saúde. Porto: FCDEF; 2003.

30. Dencker M, Thorsson O, Karlsson MK, Linden C, Eiberg S, Wollmer P, et al. Gender differences and determinants of aerobic fitness in children aged 8-11 years. Eur J Appl Phys 2007;99(1):19-26.
Endereço para correspondência
Daniel Monteiro de Vilhena e Santos
Laboratório de Cineantropometria
e Gabinete de Estatística Aplicada
Faculdade de Desporto - Universidade do Porto
Rua Dr. Plácido Costa, 91
4200-450 Porto, Portugal
E-mail: danielsantos13@hotmail.com 